\title{
A stratification method based on clustering for the minimization of data masking effect in signal detection
}

\author{
Jian-Xiang Wei ${ }^{i^{*}} \mathbb{D}$, Yue Ding ${ }^{1}$, Ming $\mathrm{Li}^{2}$ and Jun Sun ${ }^{2}$
}

\begin{abstract}
Background: Data masking is an inborn defect of measures of disproportionality in adverse drug reactions (ADRs) signal detection. Many previous studies can be roughly classified into three categories: data removal, regression and stratification. However, frequency differences of adverse drug events (ADEs) reports, which would be an important factor of masking, were not considered in these methods. The aim of this study is to explore a novel stratification method for minimizing the impact of frequency differences on real signals masking.

Methods: Reports in the Chinese Spontaneous Reporting Database (CSRD) between 2010 and 2011 were selected. The overall dataset was stratified into some clusters by the frequency of drugs, ADRs, and drug-event combinations (DECs) in sequence. K-means clustering was used to conduct stratification according to data distribution characteristics. The Information Component (IC) was adopted for signal detection in each cluster respectively. By extracting ADRs from drug product labeling, a reference database was introduced for performance evaluation based on Recall, Precision and F-measure. In addition, some DECs from the Adverse Drug Reactions Information Bulletin (ADRIB) issued by CFDA were collected for further reliability evaluation.
\end{abstract}

Results: With stratification, the study dataset was divided into 21 clusters, among which the frequency of DRUGs, ADRs or DECs followed the similar order of magnitude respectively. Recall increased by 34.95\% from 29.93 to $40.39 \%$, Precision reduced by $10.52 \%$ from 54.56 to $48.82 \%$, while F-measure increased by $14.39 \%$ from 38.65 to $44.21 \%$. According to ADRIB after 2011, 5 DECs related to Potassium Magnesium Aspartate, 61 DECs related to Levofloxacin Hydrochloride and 26 DECs related to Cefazolin were highlighted.

Conclusions: The proposed method is effectively and reliably for the minimization of data masking effect in signal detection. Considering the decrease of Precision, it is suggested to be a supplement rather than an alternative to nonstratification method.

Keywords: Adverse drug reaction, Signal detection, Data masking, Stratification, Clustering

\section{Background}

Spontaneous reporting system (SRS) is one of critical data resources for adverse drug reactions (ADRs) surveillance. Tau $\mathrm{N}$ et al. demonstrated that the most frequent information sources that served as the basis of the initial safety signal in the Unite States were Food and Drug Administration's adverse event reporting system $(87$ [38\%]) and randomized clinical trials $(81[36 \%])$ or observational

\footnotetext{
* Correspondence: jxwei@njupt.edu.cn

${ }^{1}$ School of Internet of Things, Nanjing University of Posts and

Telecommunications, Nanjing 210003, China

Full list of author information is available at the end of the article
}

studies among the 228 drug safety communications [1]. In China, medical institutions, pharmaceutical manufacturers and patients report adverse drug events (ADEs) through SRS in the voluntary reporting approach. Each report is assessed by experts of pharmacovigilance before being recorded into the Chinese Spontaneous Reporting Database (CSRD). Up to now, the number of new reports has exceeded one million per year. An important task for pharmacovigilance is to discover potential risks for postmarketing drugs by signal detection based on the CSRD. As the quality of reports varies greatly, all reports of SRS

C The Author(s). 2020 Open Access This article is distributed under the terms of the Creative Commons Attribution 4.0 International License (http://creativecommons.org/licenses/by/4.0/), which permits unrestricted use, distribution, and reproduction in any medium, provided you give appropriate credit to the original author(s) and the source, provide a link to the Creative Commons license, and indicate if changes were made. The Creative Commons Public Domain Dedication waiver (http://creativecommons.org/publicdomain/zero/1.0/) applies to the data made available in this article, unless otherwise stated. 
are mainly used for hypothesis generation of suspicious signals rather than evidence.

The conventional methods of ADR signal detection are mainly based on disproportionality analyses [2], such as Proportional Reporting Ratio (PRR), Reporting Odds Ratio (ROR), the integrated standard method taken by Medicines and Healthcare Products Regulatory Agency (MHRA), Information Component (IC), Multi-item Gamma Passion Shrinker (MGPS) and so on [3-11]. Although these methods have achieved acceptable performance [12, 13], they are strongly affected by several biases, such as under-reporting, misdiagnosis and selective reporting [14, 15], which may lead to data masking effect [16-18] or competition bias [15, 19].

Data masking is a collateral effect of quantitative methods in signal detection, which relies on disproportionality analysis by which signals of suspected drugevent combinations (DECs) may be delayed or hindered because of the over-reporting of another DEC [20]. The previous researches for minimizing data masking can be classified into three categories: data removal, regression and stratification method. In data removal method, some specific data such as the known DECs [19] and reports related to drug competitors [21] were removed to correct for competition bias and highlight suspected DECs of interest. Some reasonable statistical decision rules were proposed to determine the type and quantity of data to be removed more objectively [22]. Arnaud et al. identified [15] potential competitors via competition index, as well as masking factor [23] and masking ratio [20] and performed signal detection after removing reports mentioning such competitors. In general, the performance of data removal methods is highly dependent on human decision and rule-making. Different from data removal methods, Caster et al. [24, 25] applied lasso logistic regression into ADR surveillance and highlighted more DECs signals related to specific drugs earlier than the IC method. Each report was treated as observation object $[16,26]$ to avoid losing data, however the computation process was extremely tedious and timeconsuming. Furthermore, some researchers thought that ADRs were mostly related to drugs' medicinal properties, but the confounders of patients could not be simply ignored (e.g., age, gender, region), which would result in many false signals [11, 27, 28]. Ye et al. [29, 30] stratified the whole dataset into several strata according to suspected confounders and performed signal detection separately. However, it should be noted that confounding could only be evaluated in the absence of effect modification $[28,31]$, otherwise the integrity of data would be destroyed and false signals might come.

These adjusted methods for signal detection are mainly based on measures of disproportionality, in which suspected DECs signals are highlighted by disproportionate observed-to-expected (OE) ratios. The OE ratios are strongly affected by over-reported drugs or ADRs, and some specific DECs corresponding to true signals which are rarely reported may be masked with lower $\mathrm{OE}$ ratios. Therefore, frequency difference of ADEs is an important factor of data masking, which has not been considered in the above methods. It is reasonable to stratify the data into some clusters, among which the data is of similar order of magnitude. The aim of this study is to explore a novel stratification method to reduce the impact of frequency differences on true signals masking.

\section{Method}

\section{Data source}

All reports of ADEs in the CSRD between 1 January 2010 and 31 December 2011 were selected. By preprocessing, a study dataset including 1,081,898 records was obtained, which included 1763 drugs, 877 ADRs and 37, 193 DECs.

A reference database was considered as the gold standard for performance evaluation, which contained ADRs extracted from drug product labeling manually. If some DECs exist in the reference database but are not detected as positive signals by disproportionation analysis, we suppose these DECs are masked. Among 37,193 DECs from the CSRD, there are 12,493 DECs existing in the reference database and we denote them as known DECs.

\section{Stratification strategy}

Disproportionality analysis method, such as IC, is based on an OE ratio comparing the relative reporting rate of the ADR for a specific drug with that for the overall drugs in the database. If the usage quantities of drugs are equivalent, $\mathrm{OE}$ ratio may be more reliable. In a sense, commonly used drugs are of more reports. For example, among 1763 drugs in our study dataset, Levofloxacin Hydrochloride and Azithromycin, the two widely used drugs, were reported 111,335 times and 78,449 times, accounting for 6.18 and $4.35 \%$ of all reports respectively. Thus, the frequency of reports in SRS reflects the usage quantities of drugs indirectly.

We scanned the study dataset by the IC method and compared all suspected signals with the reference database. Table 1 revealed that the average frequency of reports on all drugs was 1022.06 times, while the average frequency of reports on the drugs related to masked DECs was 366.96 times with frequency decline rate $64.10 \%$. The signals of drugs with less frequency were more likely to be masked, just as Maignen et al. mentioned that the strongest masking effect was associated with the drug with the highest number of records for any event [20]. Similarly, overall averages on ADRs and 
Table 1 Statistics of ADE reports

\begin{tabular}{llll}
\hline & Average frequency of reports on DRUGs & Average frequency of reports on ADRs & Average frequency of reports on DECs \\
\hline Related to masked DECs & 366.96 & 1001.76 & 48.29 \\
Overall average & 1022.06 & 2054.62 & 48.45 \\
Decline rate $^{\text {a }}$ & $64.10 \%$ & $51.24 \%$ & $0.33 \%$
\end{tabular}

${ }^{\mathrm{a} D e c l i n e ~ r a t e: ~ t h e ~ p e r c e n t a g e ~ o f ~ d e c r e a s e ~ i n ~ f r e q u e n c y ~ o f ~ m a s k i n g ~ s i g n a l s ~ c o m p a r e d ~ t o ~ t h e ~ o v e r a l l ~ a v e r a g e ~}$

DECs were 2054.62 times and 48.45 times, while the corresponding average frequency of reports involved in the masked signals were 1001.76 times and 48.29 times, declined by 51.24 and $0.33 \%$ respectively. The frequency difference of drugs $(64.10 \%)$ is most obvious, followed by ADRs (51.24\%) and DECs (0.33\%). Therefore, in order to reduce the impact of frequency difference on $\mathrm{OE}$ ratio, the stratification will be conducted in the sequence of "DRUGs-ADRs-DECs".

\section{Stratification procedure}

The stratification process can be described as follows:

Step 1: Stratify the study dataset according to the frequency distribution characteristics of DRUGs. The frequency of ADR reports is counted for each drug and $K$-means clustering algorithm is adopted to partition the study data into several clusters. Cluster refers to a group of objects with the similar characteristic, and in this case, it refers to a group of drugs with similar order of magnitude in frequency.

Step 2: Further divide each cluster into multiple small clusters based on the frequency of ADRs.

Step 3: Conduct repetitive operations based on DECs subsequently to divide the study dataset into many smaller clusters.

Specifically, a cluster is divided into some small clusters in each stratification by following processes: analyze data distribution, determine the number of clusters and perform stratification with $K$-means algorithm. K-means is a clustering algorithm frequently used in data mining. It aims to partition $m$ objects into $k$ clusters, in which each object has the similar attributes. $k$ is a parameter that needs to be predefined, representing the number of clusters. First, $k$ cluster centers are randomly selected from all objects. The remaining objects are assigned to the different cluster based on the similarity measure between the object and all cluster centers. Then, cluster centers are updated by computing the mean of the objects in the same cluster. All objects are arranged into new clusters with this iterative refinement technique. Considering that the intensive areas in the data distribution chart will form peaks, $k$ is determined by peaks number of data distribution in this study.

\section{Signal detection method}

The IC method is adopted for signal detection. The lower limit of the $95 \%$ confidence interval is referred to as $\mathrm{IC}_{025}$, which is the standard measure used to screen the WHO database for excessive ADR relative reporting rates [27]. The signal with the threshold at $\mathrm{IC}_{025}>0$ is considered suspected.

\section{Performance evaluation}

Three classic indicators are adopted for performance evaluation, including Precision, Recall and F-measure [32]. Precision is a measure of exactness, indicating the percentage of DECs labeled as positive that are actually ADRs. Recall is a measure of completeness, indicating the percentage of DECs corresponding to ADRs that are labeled as positive. There tends to be an inverse relationship between Precision and Recall, where it is possible to increase one at the cost of reducing another. An alternative compromise is $F$-measure, which is the harmonic mean of Precision and Recall. According to the reference database, true positive (TP) represents the number of known DECs accurately detected as positive signals, false negative (FN) represents the number of known DECs detected as negative signals, false positive (FP) represents the number of unknown DECs detected as positive signals and true negative (TN) represents the number of unknown DECs detected as negative signals. Based on $\mathrm{TP}, \mathrm{FP}, \mathrm{FN}$ and TN, the three indicators are calculated for comparing the performance differences between stratification and non-stratification.

Meanwhile, some ADEs of the Adverse Drug Reaction Information Bulletin (ADRIB) after 2011 issued by CFDA are collected for reliability evaluation.

\section{Results \\ Stratification results}

To determine the numbers of clusters in each step, statistical analysis was adopted on data distribution. In the first step of stratification, we found the frequency of different drugs varied dramatically. For example, $\omega$-3 Fish Oil Fat Emulsion and Bicalutamide were reported only three times, while Levofloxacin Hydrochloride was up to 111,335 times. The statistic analysis of data distribution would not be obvious on account of the large data range. The natural logarithm $(l n)$ was introduced to compress the scales. The frequency ranging from 3 to 111,335 was transformed into an interval [1.1, 
11.6], and the frequency statistics was performed with an interval step of 0.5 .

The frequency histogram of 1763 drugs was presented in Fig.1. The $l n$-adjusted frequency intervals labeled the horizontal axis, and quantity of drugs marked the vertical axis. There were three peaks in Fig. 1, and each peak indicated that the data was concentrated in corresponding magnitude range. Therefore, the cluster number based on DRUGs was set as 3, and K-means clustering algorithm was performed by SPSS 19.0. Similar operations were conducted based on ADRs and DECs in sequence.

With stratification, the study dataset was eventually divided into 21 clusters, among which the frequency of DRUGs, ADRs or DECs followed the similar order of magnitude respectively. Taking DECs as an example, the frequency distribution of each cluster was illustrated by a box and whisker diagram (Fig. 2). The vertical axis represented the $l n$-adjusted frequency of DECs. The horizontal axis represented the cluster ID, which meant the hierarchical relationship among clusters. For example, the ID 1-1-1 indicated DRUGs cluster $1 \rightarrow$ ADRs cluster $1 \rightarrow$ DECs cluster 1 .

The maximum value and minimum value of each cluster were mostly in the similar order of magnitude. However, there were a few outliers in some clusters, such as cluster 3-2-3 and cluster 3-3-3. The reason for the existence of outliers was that the relatively low-frequency DECs occupied a large proportion.

\section{Performance for unmasking}

Using the IC method, 6853 suspected signals including 3739 known DECs were detected with non-stratification, and 10,336 suspected signals including 5046 known DECs were detected with stratification. Detailed results were shown in Table 2, where the values in brackets were the results of non-stratification.

With stratification, the increase in TP was 1307, while the increase in FP was 2176. Table 3 showed that Recall increased by $34.95 \%$ from 29.93 to $40.39 \%$, Precision reduced by $10.52 \%$ from 54.56 to $48.82 \%$ and $F$-measure increased by $14.39 \%$ from 38.65 to $44.21 \%$. The considerable improvement of $F$-measure confirmed the effectiveness of the proposed method.

A precision-recall curve was introduced to evaluate the overall performance of the method. The study dataset was sorted in descending order based on the $\mathrm{IC}_{025}$ values for stratification and non-stratification, and Precision and Recall for each DEC were calculated gradually. Figure 3 showed that Recall of non-stratification was slightly better than that of stratification under the same Precision at the beginning of signal detection. With more and more TP signals were detected subsequently, Recall of stratification was significantly better than that of nonstratification. On the whole, under the same Precision, Recall of stratification was better than that obtained without stratification, which proved that the performance of signal detection had improved with stratification.

For further reliability evaluation, we selected out some DECs from the ADRIB after 2011 issued by CFDA which contained true signals that were not present in the reference database. The DECs were not detected as positive signals by non-stratification, but newly highlighted by stratification. As listed in Table 4, some ADEs related to Potassium Magnesium Aspartate (in the 50th ADRIB,

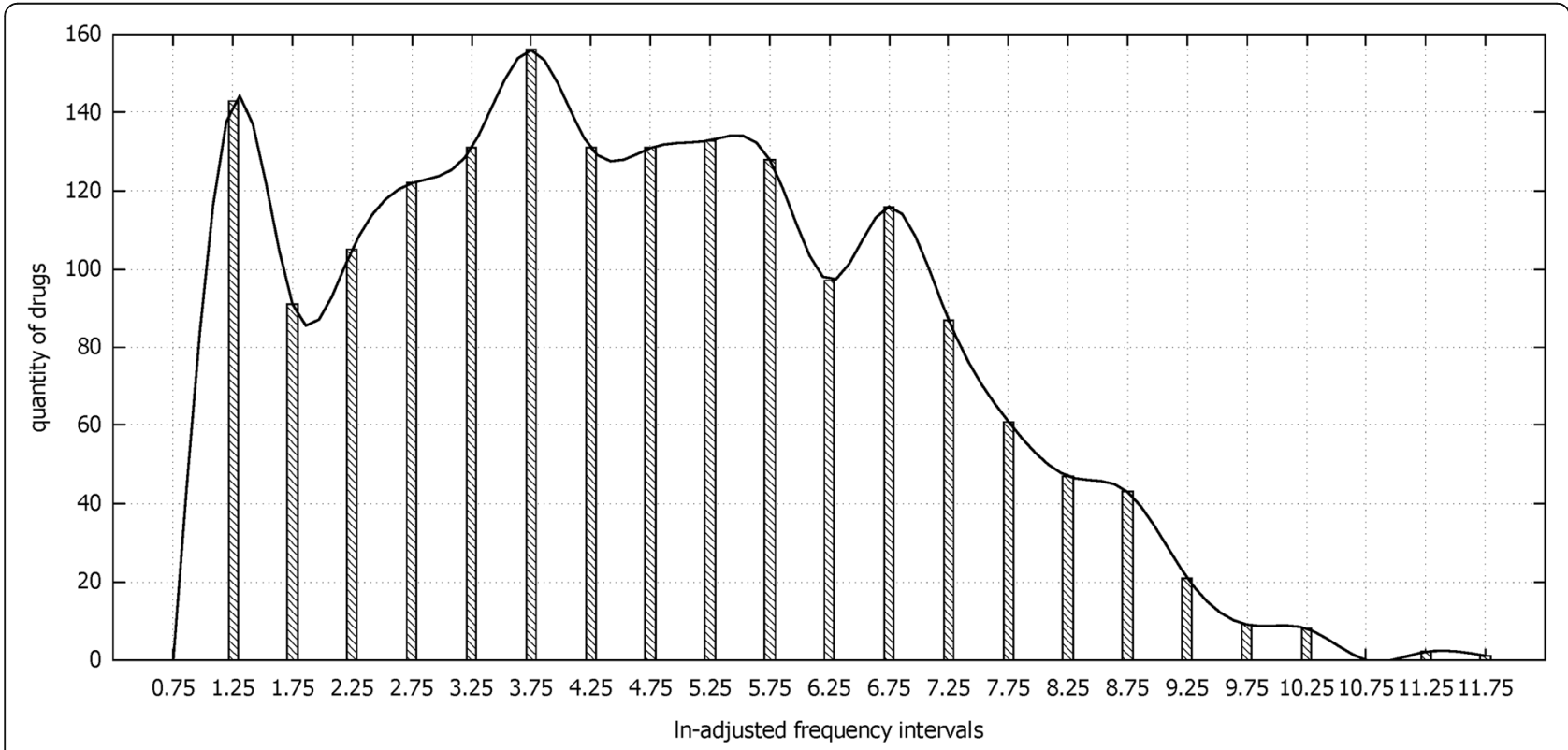

Fig. 1 Frequency distribution of drugs 


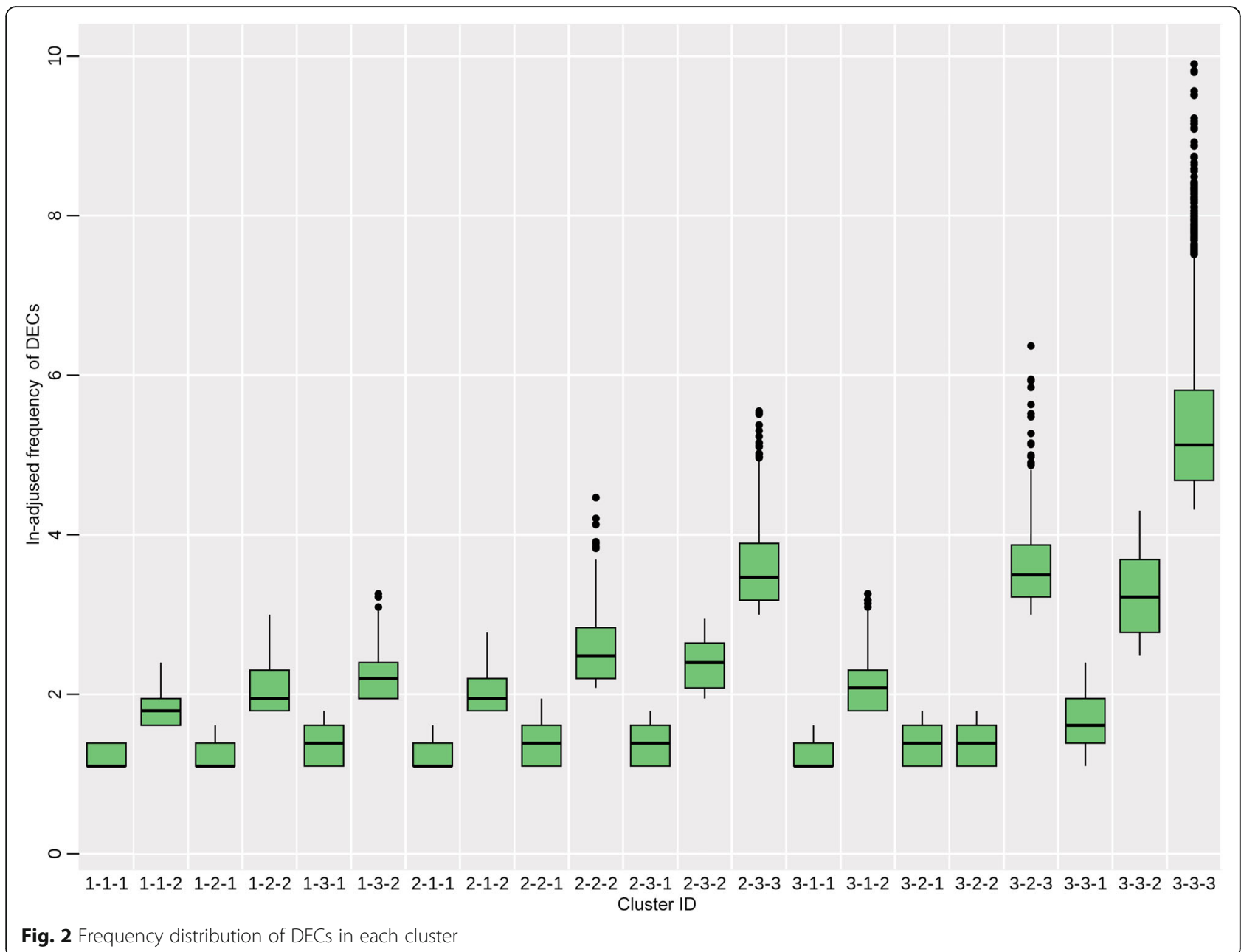

September 19, 2012) [33], Levofloxacin Hydrochloride (in the 56th ADRIB, August 2, 2013) [34] and Cefazolin (in the 59th ADRIB, January 26, 2014) [35] were detected with $\mathrm{IC}_{025}$ by stratification method. It could be noted that these DRUGs were reported in a large order of magnitude, but the frequencies of the DECs corresponding to them were very small. Therefore, when OE ratios were calculated based on disproportionality method, the expected value increased while the observed value decreased, which resulted in the decreased $\mathrm{OE}$ ratios and masked signals. Data masking effect was particularly evident to Levofloxacin Hydrochloride in Table 4. By stratification, the reports of the drug were scatter into many

Table 2 Signal detection results of stratification and nonstratification

\begin{tabular}{lcl}
\hline & Positive \\
& $\begin{array}{l}\text { Stratification (Non- } \\
\text { stratification) }\end{array}$ & $\begin{array}{l}\text { Negative } \\
\text { Stratification (Non- } \\
\text { stratification) }\end{array}$ \\
\hline Known & $5046(3739)$ & $7447(8754)$ \\
Unknown & $5290(3114)$ & $19,410(21,586)$ \\
\hline
\end{tabular}

clusters, in which the frequency declined significantly. As a result, $61 \mathrm{DECs}$ related to it were newly unmasked. Similarly, 5 DECs related to Potassium Magnesium Aspartate and 26 DECs related to Cefazolin were unmasked by stratification.

\section{Discussion}

Data masking or competition bias is an inborn defect in disproportionality analysis which depends on $\mathrm{OE}$ ratio to highlight DECs. Some measures can be adjusted to minimize any undue influence on the ADR reporting rate of covariates by performing stratification according to a set of common potential confounders [28]. However, these adjusted methods still result in data masking as ignoring frequency differences between ADEs. To reduce the impact on OE ratios, this pilot study mainly focuses on minimizing the data masking effect in signal detection by stratification based on clustering. The study dataset is stratified into some clusters according to the sequence of "DRUGs-ADRs-DECs" and signal detection is conducted by the IC method of disproportionality for each cluster respectively. All highlighted DECs are 
Table 3 Performance evaluation of stratification and nonstratification

\begin{tabular}{llll}
\hline & Precision & Recall & $F$ \\
\hline Non-stratification & $54.56 \%$ & $29.93 \%$ & $38.65 \%$ \\
Stratification & $48.82 \%$ & $40.39 \%$ & $44.21 \%$ \\
\hline
\end{tabular}

collected to evaluate unmasking performance of stratification based on the reference database and ADRIB. The specific number of clusters is determined by data distribution characteristics, and stratification is performed by K-means clustering algorithm step by step. Such processes can avoid the subjective decision existing in other stratification methods.

In our study dataset, there are more than one million reports where the frequencies of DECs vary greatly for various reasons, such as the frequent uses of drugs, the differences of drug side effects or even the individual selective reporting. The over-reported DRUGs, ADRs and DECs are more likely to mask some specific DECs which are less reported but actually true signals. The frequency distribution of reports in each cluster is smoothed by stratification, which is different from other stratification methods where the whole dataset is stratified into several strata according to confounding factors such as gender, age, region, etc.
TP signals increase from 6853 to 10,336 with stratification, which means a significant increase in the number of positive signals. These signals include 5046 TP signals and 5290 FP signals. The increase in the signals identified by our method is due to the fact that all high frequency drugs or ADEs are divided into different clusters, which reduces the possibility of the related low frequency DECs being masked. Among 5046 TP signals, 1656 signals (32.82\%) are not detected by non-stratification, which fully proves our method can better minimize data masking. While FP signals increase from 3114 to 5290 , which means more workload is need in signal evaluation for pharmacovigilance.

There are some limitations in this study. First, 2 years of spontaneous reporting data may not fully represent total data in CSRD. Then, as the gold standard for performance evaluation of signal detection, the reference database is extracted from drug product labeling manually, and the omissions or errors are unavoidable. Meanwhile, only the IC method is adopted for signal detection. The other methods of disproportionality analysis, such as PRR and MHRA, are not tried to verify the proposed method. These limitations above may lead to uncertain impact on the experimental results.



Fig. 3 Precision-recall curve 
Table 4 Signal detection results related to Potassium Magnesium Aspartate, Levofloxacin Hydrochloride and Cefazolin

\begin{tabular}{|c|c|c|c|c|}
\hline Drug (frequency) & ADR & DECs' frequency & $\mathrm{IC}_{025}$ of non-stratification & $\mathrm{I}_{025}$ of stratification \\
\hline \multirow[t]{5}{*}{ Potassium Magnesium Aspartate (2043) } & pruritus & 65 & -2.42 & 1 \\
\hline & anaphylactoid reaction & 63 & -0.67 & 0.94 \\
\hline & headache & 54 & -1.17 & 0.64 \\
\hline & dyspnoea & 40 & -0.25 & 0.48 \\
\hline & cyanosis & 11 & -0.59 & 0.06 \\
\hline \multirow[t]{10}{*}{ Levofloxacin Hydrochloride $(111,335)$} & larynx oedema & 69 & -0.32 & 4.04 \\
\hline & Infusion reaction & 66 & -0.93 & 3.76 \\
\hline & skin discolouration & 41 & -3.46 & 3.7 \\
\hline & vesicular rash & 54 & -1.46 & 3.02 \\
\hline & tachycardia & 66 & -2.37 & 2.53 \\
\hline & agitation & 75 & -0.52 & 2.37 \\
\hline & asthma & 29 & -2.2 & 2.3 \\
\hline & application site pain & 114 & -0.39 & 2.04 \\
\hline & arrhythmia & 26 & -3.04 & 1.61 \\
\hline & ecphysesis & 121 & -1.18 & 1.38 \\
\hline \multirow[t]{10}{*}{ Cefazolin $(15,105)$} & injection site rash & 67 & -0.12 & 2.27 \\
\hline & larynx oedema & 18 & -0.21 & 1.47 \\
\hline & ecphysesis & 44 & -0.17 & 1.41 \\
\hline & injection site pruritus & 30 & -1.95 & 1.39 \\
\hline & haematuria & 22 & -0.99 & 1.07 \\
\hline & oedema periorbital & 31 & -0.61 & 1.06 \\
\hline & anaesthesia local & 57 & -1.21 & 1.05 \\
\hline & cutireaction & 43 & -1.18 & 1.04 \\
\hline & diarrhoea & 72 & -3.34 & 0.9 \\
\hline & sweating increased & 79 & -0.48 & 0.9 \\
\hline
\end{tabular}

\section{Conclusion}

This paper proposes a stratification method based on clustering for the minimization of masking in signal detection. All reports of 2 years in the CSRD are stratified into some clusters, among which DRUGs, ADRs or DECs are of the similar order of magnitude in frequency. Experimental results show that better performance for unmasking signals is obtained with stratification. Owing to the decline of Precision, we suggest that this method can be used in parallel to non-stratification method rather than replacing it.

\section{Abbreviations}

ADEs: Adverse drug events; ADRIB: Adverse drug reaction information bulletin; ADRs: Adverse drug reactions; CFDA: China food and drug administration; CSRD: Chinese spontaneous reporting database; DECs: Drugevent combinations; FN: False negatives; FP: False positives; IC: Information component; MHRA: Medicines and Healthcare Products Regulatory Agency; OE: Observed-to-expected; PRR: Proportional reporting ratio; ROR: Reporting odds ratio; SRS: Spontaneous reporting system; TN: True negatives; TP: True positives

\section{Acknowledgments}

Thanks to the CFDA for providing data necessary for this research.

\section{Authors' contributions}

JXW conceived of the study. YD participated in data processing. JXW and YD participated in data analysis, and drafting of the manuscript. JS and ML were involved in critically revising the manuscript and reviewing experiment results. All authors gave final approval of the submitted manuscript, and agreed to be accountable in all aspects for the submitted manuscript.

\section{Funding}

This study was funded by National Social Science Foundation of China (14BTQ036).

\section{Availability of data and materials}

This research comes from a project which the CFDA commissioned me and other authors to undertake. All ADR spontaneous reporting data in this study is licensed by the CFDA. The data is not publicly available due to the policy of confidentiality of the CFDA but are available from the corresponding author on reasonable request and with permission of the CFDA.

\section{Ethics approval and consent to participate}

For all studies mentioned in this manuscript, no approval of the ethical review board was needed according to "Ethical Review Involving Human Biomedical Research" (No.11 Order of National Health and Family Planning Commission of China, http://www.gov.cn/gongbao/content/ 2017/content_ 5227817. html); only human subject research with a high impact for patients have to be reviewed. 


\section{Consent for publication}

Not applicable.

\section{Competing interests}

The authors declare that they have no competing interests.

\section{Author details}

${ }^{1}$ School of Internet of Things, Nanjing University of Posts and Telecommunications, Nanjing 210003, China. 2Jiangsu Center for ADR Monitoring, Nanjing 210002, China.

Received: 13 August 2018 Accepted: 28 January 2020 Published online: 03 February 2020

\section{References}

1. Tau N, Shochat T, Gafter-Gvili A, Tibau A, Amir E, Shepshelovich D. Association between data sources and US Food and Drug Administration drug safety communications. JAMA Intern Med. Published online September. 2019;03. https://doi.org/10.1001/jamainternmed.2019.3066.

2. Finney DJ. Systemic signalling of adverse reactions to drugs. Methods Inf Med. 1974;13(1):1-10.

3. Evans SJW, Waller PC, Davis S. Use of proportional reporting ratios (PRRs) for signal generation from spontaneous adverse drug reaction reports. Pharmacoepidemiol Drug Saf. 2001;10(6):483.

4. Wilson AM, Thabane L, Holbrook A. Application of data mining techniques in pharmacovigilance. Br J Clin Pharmacol. 2004:57(2):127-34.

5. Van PE, Diemont W, Van GK. Application of quantitative signal detection in the Dutch spontaneous reporting system for adverse drug reactions. Drug Saf. 2003;26(5):293-301.

6. Bate $A$, Lindquist $M$, Edwards $I R$, Orre R. A data mining approach for signal detection and analysis. Drug Saf. 2002;25(6):393-7.

7. Hauben M, Zhou X. Quantitative methods in pharmacovigilance: focus on signal detection. Drug Saf. 2003;26(3):159-86.

8. Bate A, Lindquist M, Edwards IR, Olsson S, Orre R, Lansner A, et al. A Bayesian neural network method for adverse drug reaction signal generation. Eur J Clin Pharmacol. 1998;54(4):315-21.

9. Orre R, Lansner A, Bate A, Lindquist M. Bayesian neural networks with confidence estimations applied to data mining. Comput Stat Data Anal. 2000;34(4):473-93.

10. Bate A. Bayesian confidence propagation neural network. Drug Saf. 2007; 30(7):623-5.

11. Dumouchel W. Bayesian data Mining in Large Frequency Tables, with an application to the FDA spontaneous reporting system. Am Stat. 1999:53(3): 177-90.

12. Subeesh $\mathrm{V}$, Singh $\mathrm{H}$, Maheswari $\mathrm{E}$, Beulah E. Novel adverse events of vortioxetine: a disproportionality analysis in USFDA adverse event reporting system database. Asian J Psychiatr. 2017;30:152.

13. Kim S, Park K, Kim MS, Yang BR, Choi HJ, Park BJ. Data-mining for detecting signals of adverse drug reactions of fluoxetine using the Korea adverse event reporting system (KAERS) database. Psychiatry Res. 2017;256:237.

14. Moore P, Burkhart K. Adverse drug reactions in the ICU// critical care toxicology; 2016

15. Arnaud M, Salvo F, Ahmed I, Robinson P, Moore N, Bégaud B, et al. A method for the minimization of competition Bias in signal detection from spontaneous reporting databases. Drug Saf. 2016;39(3):251-60.

16. Gould AL. Practical pharmacovigilance analysis strategies. Pharmacoepidemiol Drug Saf. 2003;12(7):559-74

17. Hauben M, Madigan D, Gerrits CM, Walsh L, Van Puijenbroek EP. The role of data mining in pharmacovigilance. Expert Opin Drug Saf. 2005;4(5):929-48.

18. Ewans SJW. Statistics: analysis and presentation of safety data. In: Talbot J, Waller P, editors. Stephens' Detection of New Adverse Drug Reactions, 5th edn. Chichester: Wiley; 2004. p. 301-28.

19. Pariente A, Didailler M, Avillach P, Miremont-Salamé G, Fourrier-Reglat A, Haramburu F, et al. A potential competition bias in the detection of safety signals from spontaneous reporting databases. Pharmacoepidemiol Drug Saf. 2010;19(11):1166-71.

20. Maignen F, Hauben M, Hung E, Holle LV, Dogne JM. A conceptual approach to the masking effect of measures of disproportionality. Pharmacoepidemiol Drug Saf. 2014;23(2):208-17.

21. Montastruc F, Salvo F, Arnaud M, Bégaud B, Pariente A. Signal of gastrointestinal congenital malformations with antipsychotics after
Minimising competition Bias: a disproportionality analysis using data from



22. Wang HW, Hochberg AM, Pearson RK, Hauben M. An experimental investigation of masking in the US FDA adverse event reporting system database. Drug Saf. 2010;33(12):1117-33.

23. Juhlin K, Ye X, Star K, Norén GN. Outlier removal to uncover patterns in adverse drug reaction surveillance-a simple unmasking strategy. Pharmacoepidemiol Drug Saf. 2013;22(10):1119-29.

24. Caster O, Madigan D, Bate A. Large-scale regression-based pattern discovery: the example of screening the WHO global drug safety database. Stat Anal Data Min. 2010;3(4):197-208.

25. Caster O, Norén GN, Madigan D, Bate A. Large-scale regression-based pattern discovery in international adverse drug reaction surveillance; 2008.

26. Xiao-fei YE, Gang CHENG, Xiao-jing GUO, Jin-fang XU, Jia HE. Masking effect in the process of signal detection of adverse drug reaction. Chinese $\mathrm{J}$ Pharmacovigilance. 2013;10(1):33-5.

27. Norén GN, Bate A, Orre R, Edwards IR. Extending the methods used to screen the WHO drug safety database towards analysis of complex associations and improved accuracy for rare events. Stat Med. 2006;25(21): 3740-57.

28. Hopstadius J, Norén GN, Bate A, Edwards IR. Impact of stratification on adverse drug reaction surveillance. Drug Saf. 2008;31(11):1035-48.

29. Wei Q, Xiao-fei Y, Chao W, Jia H. Methods of controlling confounding factors in adverse drug reaction signal detection. Chinese $J$ Pharmacovigilance. 2010;7(3):142-4.

30. Lu Z, Xiao-fei Y, Chao W, Wei Q, Wen-min D, Jia H. Application of stratification analysis in adverse drug reaction signal detection. Chinese J Pharmacovigilance. 2011;08(3):158-60.

31. Evans SJW. Stratification for spontaneous report databases. Drug Saf. 2008; 31(11):1049-52.

32. Han J. Data Mining: Concepts and Techniques. San Francisco: Margan Kaufmann; 2005.

33. CFDA. Adverse Drug Reactions Bulletin (No. 50) Alerting Severe Allergic Response to Potassium Magnesium Aspartate Injection. 2012. http://www. sda.gov.cn/WS01/CL0078/74984.html. Accessed 19 Sept 2012.

34. CFDA. Adverse Drug Reactions Bulletin (No. 56) Beware of Severe Adverse Effects of Levofloxacin Hydrochloride Injection. 2013. http://www.sda.gov.cn/ WS01/CL0078/82939.html. Accessed 2 Aug 2013.

35. CFDA. Adverse Drug Reaction Information Bulletin (No. 59) Concerned about Severe Adverse Drug Reactions of Cefazolin Injection. 2014. http:// www.sda.gov.cn/WS01/CL0078/ 96374.html. Accessed 26 Jan 2014.

\section{Publisher's Note}

Springer Nature remains neutral with regard to jurisdictional claims in published maps and institutional affiliations.

Ready to submit your research? Choose BMC and benefit from:

- fast, convenient online submission

- thorough peer review by experienced researchers in your field

- rapid publication on acceptance

- support for research data, including large and complex data types

- gold Open Access which fosters wider collaboration and increased citations

- maximum visibility for your research: over $100 \mathrm{M}$ website views per year

At $\mathrm{BMC}$, research is always in progress.

Learn more biomedcentral.com/submissions 